\title{
Advancing interventions for children with motor restrictions
}

\author{
Bert Steenbergen \\ Radboud University, Nijmegen, the Netherlands / Australian Catholic \\ University, Melbourne, Australia
}

\begin{abstract}
The two most common congenital motor disorders Cerebral Palsy (CP) and Developmental Coordination Disorder (DCD) collectively represent about $6-10 \%$ of the child population. These disorders have profound developmental consequences for the child and place him/her at a significant disadvantage or risk, particularly in view of recent societal changes to the educational system and the health care system. Moreover, these children face challenges in daily life beyond their compromised motor ability and that are related to cognitive skills and scholastic performance. In light of current societal changes, there is a pressing need to optimize therapeutic approaches such that motor function and cognitive skills of this vulnerable group of children is promoted. The major challenge is to study and develop alternative cost-effective and evidence-based treatment methods for these children.
\end{abstract}

Keywords: motor disorders, cerebral palsy, developmental coordination disorder, intervention, co-morbidity

\section{Introduction}

Skilled motor function is a prerequisite for the performance of activities of daily living (ADL). Most children seamlessly learn the motor skills that prepare them well for later schooling achievement and participation, but many children with a congenital motor disorder fail to learn essential motor skills (such as grasping and writing), which places them at a significant disadvantage relative to their peers (Bult, Verschuren, Jongmans, Lindeman, \& Ketelaar, 2011; Imms, 2008). The two most common congenital motor disorders are Cerebral Palsy (CP) and Developmental Coordination Disorder (DCD) that collectively represent about $6-10 \%$ of the child population. These disorders have profound developmental consequences for the 
child and place him/her at a significant disadvantage or risk, particularly in view of recent societal changes to the educational system and the health care system.

In the Dutch educational system mainstream schools and special education schools exist. However, this educational system is facing a major challenge considering the recent law amendment on inclusive education ("Wet passend onderwijs") in the Netherlands, that is effective from August 1st 2014. This new law compels school boards to offer an appropriate educational curriculum to all children, including those with special needs. This change not only poses significant challenges for schools (e.g., physical adaptations to classrooms, adapted teaching material, and changes of skills and the level of expertise of the teachers), but also will have enormous implications for independence, self-efficacy, and self-esteem of children with motor disorders as they endeavour to integrate within regular schools.

Second, in the last few years the health care system has also undergone some radical changes, the most prominent ones being the demand for evidence-based medicine and the need for (cost-) effective treatments. Both aspects are high on the political agenda as health care costs have increased to unprecedented levels and more and more health care providers will only reimburse treatment programs that are evidence-based.

In light of these societal changes, there is a pressing need to optimize therapeutic approaches such that motor function and participation of this vulnerable group of children is promoted. The major challenge facing us is to study and develop alternative cost-effective and evidence-based treatment methods for children with motor disorders.

Although the last decade has seen an increase in evidence from basic movement science and (clinical) cognitive neuroscience that informs treatment, translation of this knowledge into cost-effective therapies has been unacceptably slow. Although for the population of children with motor disorders, treatment programs are available, the the developmental neurocognitive mechanisms involved in these disorders is until now not well understood.

Recent insights, based on converging evidence from our and other research groups, suggest that children with CP and DCD share a common underlying neurocognitive deficit that is associated with motor learning; a compromised ability for motor prediction (Adams, Lust, Wilson, \& Steenbergen, 2014; Caeyenberghs, van Roon, Swinnen, \& Smits-Engelsman, 2009; Steenbergen \& Gordon, 2006; Steenbergen, Jongbloed-Pereboom, Spruijt, \& Gordon, 2013; Wilmut, \& Wann, 2008; Wilson, Ruddock, Smits-Engelsman, Polatajko, \& Blank, 2013). This hypothesis has been supported by recent systematic reviews (Adams et al., 2014; Steenbergen et al., 2013; Wilson et al., 2013) where strong evidence for a deficit in the internal modelling process was shown, which is a critical aspect of motor control. Although current treatment programs predominantly use physical training 
alone (e.g., constraint induced movement therapy (CIMT) programs; Gordon, 2011), recent research in adults with acquired brain damage showed two promising new techniques to stimulate damaged networks in the brain and that can be used to train the internal model: Motor Imagery (MI; internal rehearsal of a future motor action without overt motor output) and Action Observation (AO; observation of the action performed by someone else).

Together, MI and AO form a continuum with MI being internally simulated motor action and AO being externally modelled action. MI and AO play a role in learning and re-learning complex motor tasks and share common neurophysiological networks with internal modelling (Gatti et al., 2013; Jeannerod, 2001). Training of motor skills via $\mathrm{MI}$ and $\mathrm{AO}$ intervention has been successful in adults with stroke and Parkinson's disease (Page, Levine, \& Leonard, 2007; Pelosin et al., 2010; Zimmermann-Schlatter, Schuster, Puhan, Siekierka, \& Steurer, 2008). In children with congenital motor disorders, what little attention these interventions have received has been promising, suggesting new avenues for stimulating the networks involved in motor learning and possibly a new chapter in childhood neurorehabilitation (Novak et al., 2013; Steenbergen et al., 2013; Wilson et al., 2013).

In the remainder of this chapter, the possible mechanisms responsible for impaired motor planning in $\mathrm{CP}$ will be discussed followed by avenues for treatment based on these mechanisms. Next, often observed comorbidities in these children, language and working memory, are discussed. The chapter ends with an outline of challenges for future research.

\section{Motor performance and internal models}

Recent insights suggest that a common factor accounting for motor dysfunction in CP and DCD is a deficit in motor prediction, a component of the process of internal modelling (Adams et al.,2014; Steenbergen et al., 2013; Wilson et al.,2013). Prediction is a vital aspect of motor performance (Jeannerod, 2006). Internal modelling is a fundamental concept in motor control and learning (Jeannerod, 2001, 2006; Wolpert, 1997; Wolpert \& Kawato, 1998). Since its first conceptualisation, the existence of internal models has been validated in numerous behavioural studies (Katschmarsky, Cairney, Maruff, Wilson, \& Currie, 2001; Pereira, Landgren, Gillberg, \& Forssberg, 2001; Williams et al., 2011) and formalized in computational neuroscience (Kawato, 1999). Internal forward (or predictive) models contribute to volitional control by anticipating the sensory consequences of a given movement. That is, internal models provide stability to the motor system by predicting the outcome of movements before slow, sensori-motor feedback becomes available, 
providing a means of rapid online correction (Davidson \& Wolpert, 2005; Hyde \& Wilson, 2011).

The process of forward internal modeling is depicted schematically in Figure 1. When a motor plan is initiated, the motor cortex generates a motor command that is relayed to the body via descending corticospinal tracts. An efference copy of this motor command is generated in parallel as a corollary discharge and relayed to parietal-cerebellar cortices. This efferenc copy serves as a predictive signal of the sensory consequences that can be expected based on the predicted effect of the motor command. Subsequently, a comparison is made between the predicted and actual sensory feedback in parietal association cortex. A mismatch between predictive estimates of limb position indicated by sensory feedback results in the generation of error signals such that the unfolding motor output commands are corrected. Online correction is needed when the initial movement plan is not specified accurately either because the initial model was incorrect or because of environmental changes (or perturbations). The basis for online correction is a functional loop between parietal cortex and the cerebellum that monitors forward estimates of limb position. Importantly, errors in prediction serve as a training signal for refining the accuracy of the predictive models and may form an important entry point for novel treatments such as AO and MI. This is an iterative process that is fundamental to both motor control and motor learning over repeated trials/practice (Hyde \& Wilson, 2011).

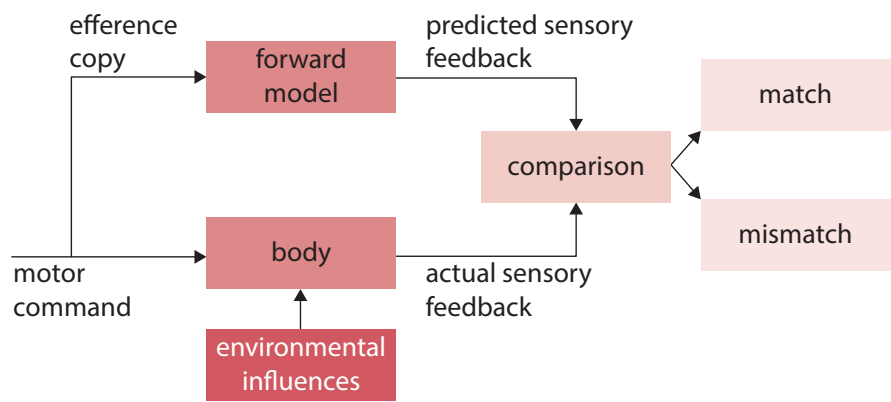

Figure 1. Forward internal modeling and prediction in motor control, based on Bubic, von Cramon, \& Schubotz (2010)

\section{Internal modelling in CP and DCD: A common deficit?}

A major challenge in understanding the nature and basis of CP and DCD is to isolate those motor control systems that might be compromised in both groups and that may explain their common motor skill learning difficulties (Gordon, Bleyenheuft, 
\& Steenbergen, 2013). Motor performance in both groups can be characterized by slow, effortful, inaccurate, and ill-coordinated movements that are overly dependent on visual feedback (Noten, Wilson, \& Steenbergen, 2014; Wilson et al., 2013). In recent years, an overwhelming amount of behavioural and neurophysiological evidence from our research group (Jongsma et al., 2015b; Steenbergen \& Gordon, 2006; Steenbergen et al., 2013; Zielinski, Jongsma, Baas, Aarts, \& Steenbergen, 2014) and others (Caeyenberghs et al., 2009; Williams et al., 2011; Wilmut \& Wann, 2008) has provided important new insights into the motor control system that is affected in these children. Studies using a range of experimental paradigms show that children with CP and DCD, despite their different aetiologies, have similar deficits in motor prediction and online control (Adams et al., 2014; Steenbergen et al., 2013; Wilson et al., 2013). Encapsulating aspects of both motor planning and feedforward control is the internal model deficit (IMD) account (Adams et al., 2014; Wilson et al., 2013). This account hypothesizes that children with CP and DCD have difficulties generating or implementing predictive models of action (Wilson \& Butson, 2007) leading to incomplete planning of a forthcoming action and a concomitant over-reliance on slower feedback-based control, which is consistent with the movement patterns seen in CP-DCD.

The IMD is displayed schematically in Figure 2 using the example of grasping a cup. On the left, the intact internal model is shown. Before the start of an action the complete movement is planned (and modeled internally), without the need for slow feedback-based control (given that no external perturbations occur). On the right, a deficit in the internal model is shown. Here, there is a reliance on (slower) sensory feedback channels at discrete points in the movement, rather than a more holistic mode of feedforward control where movement trajectory can be modulated rapidly, in flight. The result is a slow and awkward movement pattern, with numerous sub-movements.
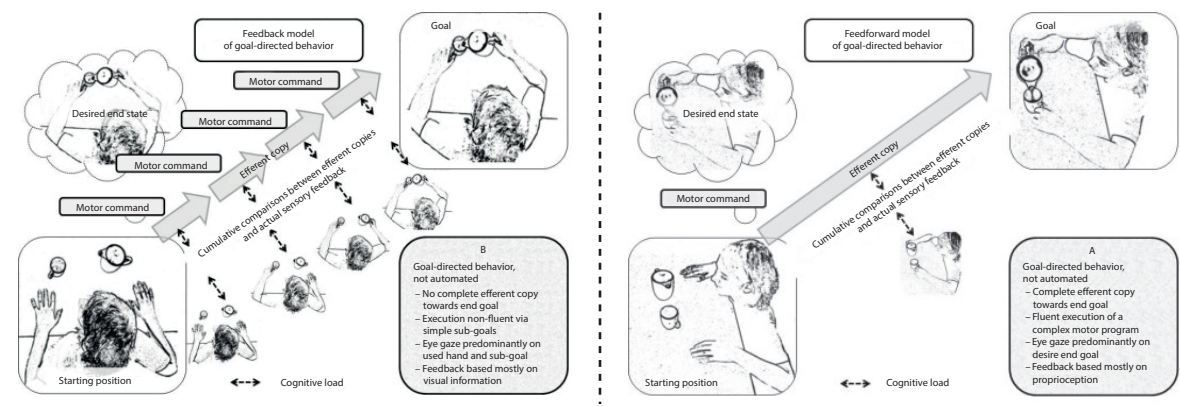

Figure 2. Daily activity of grasping a cup. Left panel: With a intact internal model. Right panel: With an internal model deficit (Jongsma, Sangen, Baas, Aarts, van der Lubbe, Meulenbroek, \& Steenbergen, 2015a) 


\section{Training the internal model: $\mathrm{A}$ innovative new treatment in $\mathrm{CP}$ and $\mathrm{DCD}$ ?}

Two well assessed strategies/modes for motor learning are Motor Imagery (MI; internal rehearsal of a motor action without overt motor output) and Action Observation (AO; observation of an action performed by someone else). Both share common aspects with the internal model and show substantial overlap on a neuroanatomical basis within the mirror neuron system (Gatti et al., 2013; Jeannerod, 2006; Vogt, Di Rienzo, Collet, Collins, \& Guillot, 2013). MI and AO are thought to be avenues by which the forward modelling process can be trained (Adams et al., 2014; Steenbergen et al., 2013; Wilson et al., 2013).

Training motor skills via MI and AO intervention have been successful in adults with brain damage such as stroke and Parkinson's disease (Dettmers, Nedelko, Hassa, Starrost, \& Schoenfeld, 2014; Ertelt et al., 2007; Kim \& Lee, 2013; Page et al., 2007; Pelosin et al., 2010; Zimmermann-Schlatter et al., 2008). In a recent study, Kim and Lee (2013) compared the additive effect of AO and MI training with physical training on the recovery from chronic stroke. Patients received a 30-min training session five times per week for a period of four weeks. They watched a video showing a person standing up, walk, and crossing obstacles (AO), and had to image the same action (MI), or received physical training. In both experimental conditions ( $\mathrm{AO}$ and $\mathrm{MI}$ ) the intervention was followed by physical training of 10 minutes. Compared to physical training alone, additional MI or AO training was shown to improve gait and mobility ability.

Experimental studies in CP and DCD have shown great promise (Buccino et al., 2012; Sgandurra et al., 2013; Wilson, Thomas, \& Maruff, 2002) for AO and MI training. For example, in a pilot study, Buccino et al. (2012) had children with CP (6-11 years) watch video clips with motor segments followed by execution of the same movement for 2 minutes. After treatment this group performed better on a motor assessment test than a control group receiving no AO training. For children with DCD, Wilson et al. (2002) showed MI training alone to yield comparable effects to physical therapy. This effect was recently replicated, indicating the strength of this intervention approach (Wilson et al., in revision, 2016). Still, research in this domain has not gone beyond this fairly descriptive level of evaluation; there is an important need for more systematic evaluation of MI and AO. 


\section{Comorbidities in CP: Factors related to language and working memory in children with Cerebral Palsy}

It is evident that children with CP and DCD face challenges in daily life and that their compromised motor ability is common denominator of these challenges. In particular in children with $\mathrm{CP}$ the mechanisms of compromised motor control have been described in quite some detail. However, the knowledge on cognitive skills and scholastic performance in these children is more restricted. In light of the high prevalence of learning problems (44\%) in children with CP (Østensjø, Carlberg, \& Vøllestad, 2003) this area warrants further investigation to advance teaching protocols for these children. In particular, the prevalence of arithmetic learning problems (28\%) and language problems (17\%) are high (Frampton, Yude, \& Goodman, 1998). Language and arithmetic are intertwined and language becomes important when arithmetic problems rely on verbal representations (Wiese, 2003). As an illustration in typically developing children, Kleemans, Segers and Verhoeven (2011) showed that phonological awareness and grammatical ability are highly related to early numeracy skills. In particular, basic knowledge of verbal representations of numbers is associated with the ability to solve simple addition and subtraction problems (Butterworth, 2005).

Studies on the factors that affect early numeracy skills in children with CP cannot claim a long research history. Jenks, de Moor and van Lieshout (2009) were among the first to examine the role of working memory for mathematical performance in children with CP. In a consecutive study, they used a wide variety of working memory tasks and found that in particular 'updating' was strongly related to mathematical achievement in children with CP that were enrolled in primary education (Jenks, van Lieshout, \& de Moor, 2012). In several follow up studies, using cross-sectional and longitudinal designs, we used structural equation modelling to scrutinize the roles of language, working memory, and motor ability for early numeracy in young children with CP. Dating back to Piaget's sensorimotor stages, leading to current approaches of embodied cognition, we hypothesized that the restricted motor capacity of children with $\mathrm{CP}$ would negatively affect their early numeracy performance. In one study we examined the extent to which cognitive and motor factors were related to arithmetic performance of a large group of primary school children with CP (Van Rooijen et al., 2012). Factors that were tested were non-verbal intelligence, working memory, word decoding capacities, and grossand fine motor skills. It was found that word decoding and fine motor skills were the strongest predictors of arithmetic performance. Using a longitudinal design in which children from 7-9 years were followed we (Van Rooijen et al., 2014) largely confirmed the findings of the earlier study. Moreover, word decoding skills were related to the initial level of arithmetic performance, but did not contribute to the 
growth rate of arithmetic performance. This result emphasizes the importance of early language skills, not only for their own sake, but also for their contribution to learning early numeracy. This study also showed that non-verbal intelligence and working memory were factors that did contribute to growth rate. As such, they are useful and valid entry-points for remediation.

In typically developing children, Toll and Van Luit (2013) showed that a remediation program consisting of teaching basic numerical concepts and language related to math was effective in improving numeracy knowledge. In children with $\mathrm{CP}$, training of working memory and early language skills to improve early numeracy and arithmetic performance of children with CP are the most promising avenues to proceed (Van Rooijen, Verhoeven, \& Steenbergen, 2015). Future intervention studies, informed by basic results as the ones mentioned above, will lead to a further refinement of education protocols for children with CP. Ultimately it will allow us to distinguish the factors that are crucial to improve teaching efficacy of this vulnerable group of children.

\section{Challenges for the future}

It is evident that independent lines of research converge on the idea that MI and $\mathrm{AO}$ are useful tools in motor rehabilitation of individuals with brain-damage. The next step is to directly test this assumption in children with congenital motor disorders, targeting putative neural networks that are known to support motor prediction. Specifically, the development of child friendly, developmentally-appropriate, cost-effective and theoretically principled treatment programs is at the vanguard of work in paediatric rehabilitation and will lead to a targeted (and tailored) approach to intervention. In addition, research in this area will advance the much needed cost-efficacy of current rehabilitation treatments. Similarly, in an education context, the delineation of the critical factors that affect the learning of early numeracy and mathematics will improve efficacy of educational programs for children with CP.

A crucial element to advance the integration between basic research and intervention approaches is to start from an integrative multi-disciplinary and multi-level approach. Such an approach is essential for the progress of evidence-based treatment. It allows for delineation of the critical determinants for treatment success on the behavioral and neural level and their interaction. This will advance our understanding of the neurocognitive mechanisms that must be targeted to ensure treatment efficacy in the congenitally damaged brain. Another element that needs more elaborate study in the future is the heterogeneity in the population under study by taking into account individual variation in training efficacy and mediating factors at different levels (cognitive, motor, and neurophysiological). Obviously, this 
will lead to more individualized treatment, and can also inform us on the lack of treatment effects in some participants. That is, future research needs to move from group-based to more individual-based research and intervention approaches. In this respect, EEG techniques can used be to track individual progress in therapy, for example via the development of algorithms that provide critical markers of neural function along networks that support motor learning. Furthermore, the identification of children that show either more MI or more AO induced EEG activations might serve as a biological marker based on which effective training programs can be offered. This has implications for other fields such as basic neuroscience and medicine as well. With respect to the latter, these markers can be used as screening and selection tools to predict success and optimal treatment doses at an early stage in turn promoting cost-effective health care. In fact, the electroencephalogram (EEG) is an excellent method to study the brain in its natural setting and as such takes cognitive neuroscience to the next level, that is, to make a move 'outside the laboratory setting into the real world', thereby facilitating knowledge transfer.

Finally, in future research more and more emphasis will be placed on knowledge transfer. That is, researchers increasingly need to be aware of the possible transfer and implementation of their research results. This poses an extra task for researchers and not one for which they have had extensive training. For example, essential in this respect is that research programs and projects need to be carried out in close collaboration with professional partners from different disciplines (e.g., psychology, physiotherapy, pedagogy, neurology) and also patient organizations in order to establish, and test feasibility of developed interventions. This demands a different type of organization and communication than the one that is 'common ground' for researchers. In a recent document, we have outlined how such knowledge transfer might be organized within social sciences (Steenbergen, Hilhorst, Steenbergen, Gelinck, \& Boer, 2015). Key aspect of such an organization is that scientists and stakeholders from outside academics formulate research questions in 'co-creation', such that research questions, and the results will have value outside academics. Eventually, such a structured organization will aid the transfer of scientific findings and knowledge, and ensures that the outcomes will complement existing treatment services in a timely manner with respect to societal developments related to health issues and education.

\section{References}

Adams, I., Lust, J., Wilson, P., \& Steenbergen, B. (2014). Compromised motor control in children with DCD: A deficit in the internal model? - a systematic review. Neuroscience and Biobehavourial Reviews, 47, 225-244. doi:10.1016/j.neubiorev.2014.08.011 
Bubic, A., von Cramon, D. Y., \& Schubotz, R. I. (2010). Prediction, cognition and the brain. Frontiers in Human Neuroscience, 4(25).

Buccino, G., Arisi, D., Gough, P., Aprile, D., Ferri, C., Serotti, L., Tiberti, A., \& Fazzi, E. (2012). Improving upper limb motor functions through action observation treatment: a pilot study in children with cerebral palsy. Developmental Medicine and Child Neurology, 54, 822-828. doi:10.1111/j.1469-8749.2012.04334.X

Bult, M. K., Verschuren, O., Jongmans, M. J., Lindeman, E., \& Ketelaar, M. (2011). What influences participation in leisure activities of children and youth with physical disabilities? A systematic review. Research in Developmental Disabilities, 32, 1521-1529.

doi:10.1016/j.ridd.2011.01.045

Butterworth, B. (2005). The development of arithmetic abilities. Journal of Child Psychology and Psychiatry, 46(1), 3-18. doi:10.1111/j.1469-7610.2004.00374.x

Caeyenberghs, K., van Roon, D., Swinnen, S. P., \& Smits-Engelsman, B. C. (2009). Deficits in executed and imagined aiming performance in brain-injured children. Brain and Cognition, 69(1), 154-161. doi:10.1016/j.bandc.2008.07.001

Davidson, P. R., \& Wolpert, D. M. (2005). Widespread access to predictive models in the motor system: A short review. Journal of Neural Engineering, 2(3), S313-319. doi: 10.1088/1741-2560/2/3/S11

Dettmers, C., Nedelko, V., Hassa, T., Starrost, K., \& Schoenfeld, M. A. (2014). 'Video therapy': Promoting hand function after stroke by action observation training: A pilot randomized controlled trial. International Journal of Physical Medicine and Rehabilitation, 2, 189.

Ertelt, D., Small, S., Solodkin, A., Dettmers, C., McNamare, A., Binkofski, F., \& Buccino, G. (2007). Action observation has a positive impact on rehabilitation of motor deficits after stroke. Neuroimage, 36, T164-T173. doi:10.1016/j.neuroimage.2007.03.043

Frampton, I., Yude, C., \& Goodman, R. (1998). The prevalence and correlates of specific learning difficulties in a representative sample of children with hemiplegia. British Journal of Educational Psychology, 68, 39-51. doi:10.1111/j.2044-8279.1998.tbo1273.x

Gatti, R., Tettamanti, A., Gough, P. M., Riboldi, E., Marinoni, L., \& Buccino, G. (2013). Action observation versus motor imagery in learning a complex motor task: A short review of literature and a kinematics study. Neuroscience Letters, 540, 37-42. doi:10.1016/j.neulet.2012.11.039

Gordon, A. M. (2011). To constrain or not to constrain, and other stories of intensive upper extremity training for children with unilateral cerebral palsy. Developmental Medicine and Child Neurology, 53, 56-61. doi:10.1111/j.1469-8749.2011.04066.x

Gordon, A. M., Bleyenheuft, Y., \& Steenbergen, B. (2013). Pathophysiology of Cerebral Palsy and its relation to treatment. Developmental Medicine and Child Neurology, 55, 32-37.

doi: $10.1111 /$ dmcn.12304

Hyde, C., \& Wilson, P. H. (2011). Dissecting online control in Developmental Coordination Disorder: a kinematic analysis of double-step reaching. Brain and Cognition, 75(3), 232-241. doi:10.1016/j.bandc.2010.12.004

Imms, C. (2008). Children with cerebral palsy participate: A review of the literature. Disability and Rehabilitation, 30(24), 1867-1884. doi:10.1080/09638280701673542

Jeannerod, M. (2001). Neural simulation of action: A unifying mechanism for motor cognition.

Neuroimage, 14, 103-109. doi:10.1006/nimg.2001.0832

Jeannerod, M. (2006). Motor cognition. Oxford: Oxford University Press. doi:10.1093/acprof:0so/9780198569657.001.0001 
Jenks, K., de Moor, J., \& van Lieshout, E. C. D. M. (2009). Arithmetic difficulties in children with cerebral palsy are related to executive function and working memory. Journal of Child Psychology and Psychiatry, 50(7), 824-833. doi:10.1111/j.1469-7610.2008.02031.x

Jenks, K., van Lieshout, E. C. D. M., \& de Moor, J. (2012). Cognitive correlates of mathematical achievement in children with cerebral palsy and typically developing children. British Journal of Educational Psychology, 82, 120-135. doi:10.1111/j.2044-8279.2011.02034.x

Jongsma, M. L. A., Sangen, A. F. M., Baas, C. M., Aarts, P. B. M., van der Lubbe, R. H. J., Meulenbroek, R. G. J., \& Steenbergen, B. (2015a). Event-Related Potentials Elicited in a Motor Imagery Task in Children with Unilateral CP Reveal Motor Imagery Capacity with respect to the Unaffected, but not the Affected Hand. 30th International Congress of Clinical Neurophysiology (ICCN) of the IFCN (P557 - abstract book), Berlin, Germany, 20-23 March 2014.

Jongsma, M. L. A., Sangen, A. F. M., Baas, C. M., Aarts, P. B. M., van der Lubbe, R. H. J., Meulenbroek, R. G. J., \& Steenbergen, B. (2015b). Motor imagery in children with unilateral cerebral palsy: Event-related potentials demonstrate diminished motor imagery capacity for the affected hand. Developmental Medicine and Child Neurology. June 11.

doi:10.1111/dmcn.12819

Katschmarsky, S., Cairney, S., Maruff, P., Wilson, P. H., \& Currie, J. (2001). The ability to execute saccades on the basis of efference copy: impairments in double-step saccade performance in children with developmental co-ordination disorder. Experimental Brain Research, 136(1), 73-78. doi: $10.1007 /$ s002210000535

Kawato, M. (1999). Internal models for motor control and trajectory planning. Current Opinion in Neurobiology, 9(6), 718-727. doi:10.1016/So959-4388(99)0oo28-8

Kim, J.-H., \& Lee, B.-H. (2013). Action observation training for functional activities after stroke: A pilot randomized controlled trial. Neurorehabilitation, 33, 556-574.

Kleemans, T., Segers, E., \& Verhoeven, L. (2011). Cognitive and linguistic precursors to numeracy in kindergarten: Evidence from first and second language learners. Learning and Individual Differences, 21, 555-561. doi:10.1016/j.lindif.2011.07.008

Noten, M., Wilson, P., \& Steenbergen, B. (2014). The relation between motor planning and motor imagery in children with DCD. Research in Developmental Disabilities, 35, 1152-1159. doi:10.1016/j.ridd.2014.01.026

Novak, I., McIntyre, C., Morgan, C., Campbell, L., Dark, L., Morton, N., Stumbles, E., Wilson, S. A., \& Goldsmith, S. (2013). A systematic review of interventions for children with cerebral palsy: State of the evidence. Developmental Medicine and Child Neurology, 55, 885-910. doi: $10.1111 /$ dmcn.12246

Østensjø, S., Carlberg, E. B., \& Vøllestad, N. K. (2003). Everyday functioning in young children with cerebral palsy: Functional skills, caregiver assistance, and modifications of the environment. Developmental Medicine and Child Development, 45(9), 603-612.

doi:10.1111/j.1469-8749.2003.tboog64.x

Page, S. J., Levine, P., \& Leonard, A. C. (2007). Mental practice in chronic stroke: results of a radomized, placebo-controlled trial. Stroke, 38, 1293-1297.

doi:10.1161/01.STR.0000260205.67348.2b

Pelosin, E., Avanzino, A., Bove, M., Stramesi, P., Nieuwboer, A., \& Abbruzzese, G. (2010). Action observation improves freezing of gait in patients with Parkinson's disease. Neurorehabilitation and Neural Repair, 24, 746-752. doi:10.1177/1545968310368685 
Pereira, H. S., Landgren, M., Gillberg, C., \& Forssberg, H. (2001). Parametric control of fingertip forces during precision grip lifts in children with DCD (developmental coordination disorder) and DAMP (deficits in attention motor control and perception). Neuropsychologia, 39(5), 478-488. doi:10.1016/Soo28-3932(00)00132-9

Sgandurra, G., Ferrari, A., Cossu, G., Guzzetta, A., Fogassi, L., \& Cioni, G. (2013). Randomized trial of observation and execution of upper extremity actions versus action alone in children with unilateral cerebral palsy. Neurorehabilitation and Neural Repair, 27, 808-815. doi: $10.1177 / 1545968313497101$

Steenbergen, B., \& Gordon, A. M. (2006). Activity limitation in hemiplegic cerebral palsy: Evidence for disorders in motor planning (review). Developmental Medicine and Child Neurology, 48, 780-783. doi:10.1017/So012162206001666

Steenbergen, B., Hilhorst, J., Steenbergen, J., Gelinck, R., \& Boer, R. (2015). Valoriseren moet je organiseren! - Naar meer-waarde van kennis binnen de sociale wetenschappen. Discussiedocument opgesteld door Radboud Universiteit Nijmegen, Kennispraktijk en Nederlands Instituut voor Sport en Bewegen. doi:10.13140/RG.2.1.4142.6640

Steenbergen, B., Jongbloed-Pereboom, M., Spruijt, S., \& Gordon, A. M. (2013). Impaired motor planning and motor imagery in children with cerebral palsy: Challenges for the future of pediatric rehabilitation. Developmental Medicine and Child Neurology, 55, 43-46. doi: $10.1111 /$ dmcn.12306

Toll, S. W. M., \& Van Luit, J. E. H. (2013). Accelerating the early numeracy development of kindergartners with limited working memory skills through remedial education. Research in Developmental Disabilities, 34, 745-755. doi:10.1016/j.ridd.2012.09.003

Van Rooijen, M., Verhoeven, L., Smits, D-W., Ketelaar, M., Becher, J. G., \& Steenbergen, B. (2012). Arithmetic performance of children with cerebral palsy: The influence of cognitive and motor factors. Research in Developmental Disabilities, 33(2), 530-537.

doi:10.1016/j.ridd.2011.10.020

Van Rooijen, M., Verhoeven, L., Smits, D-W., Ketelaar, M., Becher, J. G., \& Steenbergen, B. (2014). Cognitive precursors of arithmetic development in primary school children with cerebral palsy. Research in Developmental Disabilities, 35, 826-832. doi:10.1016/j.ridd.2014.01.016

Van Rooijen, M., Verhoeven, L., \& Steenbergen, B. (2015). Working memory and fine motor skills predict early numeracy performance of children with cerebral palsy. Child Neuropsychology, 12, 1-13.

Vogt, S., Di Rienzo, F., Collet, C., Collins, A., \& Guillot, A. (2013). Multiple roles of motor imagery during action observation. Frontiers in Human Neuroscience, 7, a807. doi: 10.3389/fnhum.2013.00807

Wiese, H. (2003). Iconic and non-iconic stages in number development: The role of language. Trends in Cognitive Sciences, 7(9), 385-390. doi:10.1016/S1364-6613(03)00192-X

Williams, J., Anderson, V., Reddihough, D. S., Reid, S. M., Vijayakumar, N., \& Wilson, P. H. (2011). A comparison of motor imagery performance in children with spastic hemiplegia and developmental coordination disorder. Journal of Clinical and Experimental Neuropsychology, 33(3), 273-282. doi:10.1080/13803395.2010.509714

Wilmut, K., \& Wann, J. (2008). The use of predictive information is impaired in the actions of children and young adults with Developmental Coordination Disorder. Experimental Brain Research, 191(4), 403-418. doi:10.1007/s00221-008-1532-4

Wilson, P. H., Adams, I. L. J., Caeyenberghs, K., Williams, J., Thomas, P., Smits-Engelsman, B., \& Steenbergen, B. (in revision, 2016). Motor imagery training enhances motor skill in children with DCD: A replication study. Manuscript submitted for publication. 
Wilson, P. H., \& Butson, M. (2007). Deficits underlying DCD (Chapter 4). In R. H. Geuze (Ed.), Developmental Coordination Disorder: A review of current approaches (pp. 115-119). Marseille: Solal Editeurs.

Wilson, P. H., Ruddock, S., Smits-Engelsman, B., Polatajko, H., \& Blank, R. (2013). Understanding performance deficits in developmental coordination disorder: A meta-analysis of recent research. Developmental Medicine and Child Neurology, 55(3), 217-228.

doi:10.1111/j.1469-8749.2012.04436.x

Wilson, P. H., Thomas, P. R., \& Maruff, P. (2002). Motor imagery training ameliorates motor clumsiness in children. Journal of Child Neurology, 17(7), 491-498. doi:10.1177/088307380201700704

Wolpert, D. M. (1997). Computational approaches to motor control. Trends in Cognitive Science, 1(6), 209-216. doi:10.1016/S1364-6613(97)01070-X

Wolpert, D. M., \& Kawato, M. (1998). Multiple paired forward and inverse models for motor control. Neural Networks, 11(7-8), 1317-1329. doi:10.1016/So893-6080(98)ooo66-5

Zielinski, I. M., Jongsma, M., Baas, C. M., Aarts, P. B. M., \& Steenbergen, B. (2014). Unravelling Developmental Disregard in children with unilateral Cerebral Palsy by measuring Event Related Potentials during a simple and complex task. BMC Neurology, 14, 6.

Zimmermann-Schlatter, A., Schuster, C., Puhan, M. A., Siekierka, E., \& Steurer, J. (2008). Efficacy of motor imagery in post-stroke rehabilitation: a systematic review. Journal of Neuroengineering and Rehabilitation, 5, 8. doi:10.1186/1743-0003-5-8 

Created from ubnru-ebooks on 2019-09-02 04:43:06. 